\title{
Site-Specific Sensitization of Defensive Reflexes in Aplysia: A Simple Model of Long-Term Hyperalgesia
}

\author{
Edgar T. Walters \\ Department of Physiology and Cell Biology, University of Texas Medical School at Houston, Houston, Texas 77225
}

Brief, noxious, electrical or mechanical stimulation of the skin of Aplysia produces enhancement of defensive reflexes triggered at the same site for at least a week after the noxious stimulation. This site-specific behavioral sensitization can be expressed as an increase in duration of the siphon-withdrawal reflex and as an increase in magnitude of the tail-withdrawal reflex. It is unlikely that peripheral factors play a predominant role in the long-term memory. First, long-term enhancement is blocked when the CNS is disconnected from the noxious stimulation site by nerve transection. Second, long-term enhancement is blocked by preventing neural activation at the noxious stimulation site, indicating that persistent physical damage alone is insufficient to cause the enhancement. A role for activity-dependent extrinsic modulation (ADEM) of mechanosensory neurons is suggested by similar site-specific enhancement produced when weak sensory activation is paired with general modulation elicited by strong stimulation of a distant site. Because this pairing represents a form of classical conditioning, site-specific sensitization and cutaneous classical conditioning appear to be closely related in this system. These findings suggest that site-specific sensitization reflects, at least in part, a central, long-term memory of injury. This form of memory may be phylogenetically widespread, and functionally similar to aspects of hyperalgesia. In addition, the close relationship between site-specific sensitization and cutaneous classical conditioning supports the hypothesis that some forms of classical conditioning evolved from mechanisms of sensitization.

Simple defensive responses in Aplysia such as the gill-, siphon-, and tail-withdrawal reflexes have attracted considerable attention as model systems in which to study the cellular mechanisms of behavioral sensitization and classical conditioning (e.g., Castellucci et al., 1970; Carew et al., 1981; Hawkins et al., 1983; Walters and Byrne, 1983; Walters et al., 1983b). Recently, Walters and Byrne (1983) and Hawkins et al. (1983) suggested independently that classical conditioning can be produced by activity-dependent extrinsic modulation (ADEM) - a temporally

\footnotetext{
Received Mar. 10, 1986; revised July 21, 1986; accepted July 22, 1986.

This work was supported by National Institutes of Mental Health Grant MH38726, National Institutes of Health Research Career Development Award NS00848, and by the Chicago Community Trust/Searle Scholars Program. I thank L. Foster for technical assistance and Dr. J. H. Byrne for comments on an earlier draft of this paper.

Correspondence should be addressed to Dr. Edgar T. Walters, Department of Physiology and Cell Biology, University of Texas Medical School, P.O. Box 20708, Houston, TX 77225.

Copyright (C) 1987 Society for Neuroscience $0270-6474 / 87 / 020400-08 \$ 02.00 / 0$
}

specific enhancement by spike activity (intrinsic to the cell) of the effects on the cell of extrinsic neuromodulatory signals. Although ADEM of sensory neurons mediating the conditioned stimulus (CS) seems likely to be involved in the classical conditioning reported for these reflexes (e.g., Carew et al., 1981, 1983; Ingram and Walters, 1984), it has also becn implicatcd in long-term enhancement of sensory neuron connections in the isolated nervous system (Walters and Byrne, 1985). This longterm synaptic enhancement appears similar to long-term potentiation (LTP) described in other preparations. Since this and most other examples of LTP have been produced by nonassociative procedures, it seemed possible that LTP, and the mechanism of ADEM, might also be used in the intact animal for the storage of information other than temporal associations between pairs of stimuli.

In particular, ADEM in a nociceptive system might be used to encode site specificity for long-term memory of cutaneous injury. Such a role might be analogous to some of the functions of long-term hyperalgesia in man (Lewis, 1942), and of animal models of hyperalgesia involving injury-induced, site-specific alterations that can last for weeks (Woolf, 1984). This function in Aplysia is consistent with a nociceptive role of the sensory neurons (Walters et al., 1983a) shown to undergo ADEM, and in fact is predicted by the ADEM model since the sensory neurons mediating the noxious unconditioned stimulus (US) during aversive conditioning would also undergo ADEM - they would be active immediately before they triggered the general release of the sensitizing neuromodulators and should thus show changes similar to those observed in sensory neurons mediating the CS.

The present results show that defensive withdrawal reflexes in Aplysia undergo long-term, site-specific sensitization following either injury of a site or strong electrical stimulation of a site thought to produce ADEM of sensory pathways from that site (Walters and Byrne, 1983). The fact that site-specific sensitization can be produced both nonassociatively and by an explicit associative procedure supports the view that some forms of classical conditioning may have evolved from mechanisms of sensitization. Some of these results have been described in abstract form (Walters, 1985).

\section{Materials and Methods}

These studies were performed during the winter and spring on Aplysia californica (200-450 gm) supplied by Alacrity Marine Biological Services (Redondo Beach, CA). Animals were kept at $19^{\circ} \mathrm{C}$ and fed romaine lettuce in quantities sufficient to maintain a constant body weight $( \pm 10 \%)$. In all but the last experiment described below, the training and testing were conducted in a round stainless-steel bowl (diameter at water surface, $22 \mathrm{~cm}$ ) containing artificial seawater ("Instant Ocean") from the home tank. The water in the bowl was changed prior to each test, and 
its temperature did not rise above $20^{\circ} \mathrm{C}$. Each animal was gently transferred by hand into the bowl $10 \mathrm{~min}$ prior to delivery of the test stimulus. After the test stimulus the animal was observed for $5 \mathrm{~min}$ and returned to the home tank. All measurements were made "blind" by an observer who knew neither the training history of the animal nor the purpose of the experiment. The duration of siphon withdrawal was found to be the clearest index of behavioral plasticity in the unrestrained animal (see also Pinsker et al., 1973; Carew et al., 1981). Siphon-withdrawal duration was measured as the time from stimulus onset until the tip of the siphon projected again beyond both parapodia. In contrast to some studies (e.g., Pinsker et al., 1973), no cutoffs in duration were used. In some cxpcriments tail-withdrawal magnitude was estimated using an arbitrary 5-point scale.

The test stimulus was a $0.5 \mathrm{sec}$ train of weak AC shock $(10 \mathrm{~mA}, 60$ $\mathrm{Hz}$ ) applied to a restricted site by light contact with the spherical tip (diameter about $3 \mathrm{~mm}$ ) of an $\mathrm{Ag} / \mathrm{AgCl}$ monopolar electrode. To insure that the same site could be found repeatedly for testing and training, a loop of light suture thread was sewn into the skin at each selected site $3 \mathrm{~d}$ prior to the experiment. The animal was anesthetized with isotonic $\mathrm{MgCl}_{2}$ during this implantation. The only sites not marked were the tentacles, the tips of which could be identified reliably. On the first morning of each study, 2 baseline tests (1 on each side of the animal, separated by at least $90 \mathrm{~min}$ ) were given prior to training. Each site was tested once again in the afternoon. On subsequent days, only the morning tests were given.

The training stimulus in most experiments was a $45 \mathrm{sec}$ sequence of intense AC shock (50-70 mA) applied through hand-held dual capillary electrodes (diameter of each tube, $5 \mathrm{~mm}$ ) filled with artificial seawater. This wider electrode allowed focal delivery of a large current to each site, sufficient to cause strong, general defensive responses, without causing obvious tissue damage. The general defensive responses included the release of ink and opaline, locomotion and long siphon withdrawals (2-10 min). Animals that failed to release ink during training were dropped from the study. In most experiments the training stimulus was delivered to a site on the tail, but sites on the midbody and head were also used (Figs. 1, 6). The temporal parameters of the training stimulus were similar to those used previously to induce long-term enhancement of monosynaptic connections in the tail-withdrawal circuit of Aplysia (Walters and Byrne, 1985) and LTP in some mammalian preparations (e.g., Levy and Steward, 1983; Berger, 1984). Specifically, ten $0.5 \mathrm{sec}$ trains of shock $(60 \mathrm{~Hz} \mathrm{AC})$ were delivered at $5 \mathrm{sec}$ intervals. Training was conducted in a square plastic tub, separate from the stainless steel bowl used for testing.

Most of the experiments involved changes in response caused by 2 different training conditions (trained vs untrained, or paired vs unpaired) applied to each animal, and were designed to test 2 explicit hypotheses: (1) that site-specific reflex enhancement would occur at a retention interval ( $2 \mathrm{hr}$ ) when "long-term enhancement" of sensory neuron connections had been observed following analogous "training" of the isolated CNS (Walters and Byrne, 1985), and (2) that site-specific reflex enhancement would last for close to a week (4-7 d), a retention period observed previously for long-term general sensitization in Aplysia (Pinsker et al., 1973; Frost et al., 1985). These hypotheses were evaluated using paired procedures $(t$ test for correlated means or Wilcoxon's signedrank test). Because tail-withdrawal measures were made using a discrete scale, $t$ tests on these data included a correction for continuity (Snedecor and Cochran, 1980). Comparisons that were not based upon the results of preliminary pilot experiments are explicitly noted, and were tested with 2-tailed procedures. All other comparisons were 1-tailed. Several of the groups displayed an apparent positive skew in their response distributions. These samples were too small for traditional tests of skewness (Snedecor and Cochran, 1980), but it was desirable to have an objective criterion by which to apply parametric or nonparametric statistics. An arbitrary criterion was based on the proximity of the median to the midpoint of the range (a crude index of skewness). In a normal distribution the median is near the midpoint and the "skew ratio" (maximum - median)/(median - minimum) is close to 1 . It was decided that groups having a "skew ratio" differing from 1 by a factor of 2 or more would be considered to deviate from a normal distribution and would be analyzed with nonparametric statistics. In these cases, individual comparisons were made with Wilcoxon's signed-rank test if both training conditions were applied to the same animal, and with the Mann-Whitney $U$ test if the training conditions were applied to different animals. The skewed response ranges were often quite large and are reported in each figure legend.

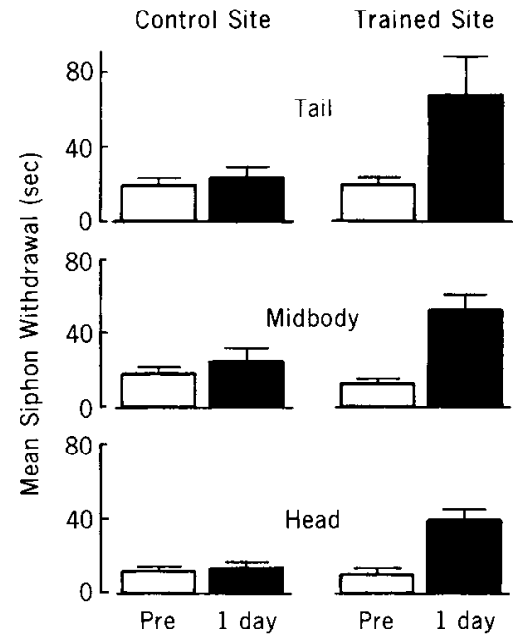

Figure 1. General and site-specific sensitization in different parts of the body. The pretraining baseline test (Pre) was given $2 \mathrm{hr}$ prior to training 1 of 2 bilateral test sites with a 45 sec sequence of strong shock. In different animals the bilateral test sites were on the dorsal surface of the tail, the side of the midbody region, or the anterior tentacles (head). When tested again $1 \mathrm{~d}$ after the training sequence, the control site showed that there was no significant general sensitization as measured by mean siphon withdrawal duration ( $₫$ SEM), while the trained site in each part of the body displayed site-specific sensitization.

\section{Results}

Site-specific sensitization of siphon withdrawal produced by electrical stimulation of different parts of the body

To test the possibility that noxious stimulation causes specific enhancement of defensive responses elicited by test stimuli applied to the same site, 3 groups of animals ( $N=4$ in each) were trained $2 \mathrm{hr}$ after a single baseline test to 2 bilateral test sites. One group received the weak test shock to the anterior tentacles ("head"), one to the midbody near the base of the parapodia (halfway down the length of the animal), and one to the side of the tail. In each case the weak test stimuli were applied to bilaterally symmetrical sites (with about 90 min between tests), and the brief, strong "training" sequence (see Materials and Methods) was applied to 1 of the 2 test sites (chosen by a coin flip). The control test site in each case showed little evidence of general sensitization when tested $1 \mathrm{~d}$ after the $45 \mathrm{sec}$ training sequence applied to the contralateral site (Fig. 1; pooling the 3 groups and comparing the $1 \mathrm{~d}$ test to the pretraining baseline, $t_{11}=0.94$, not significant). By contrast, the test stimulus applied to the trained site $1 \mathrm{~d}$ after training produced significant enhancement of the siphon-withdrawal response relative to baseline (Fig. $1 ; t_{11}=5.53 ; p<0.001$ ). In addition, within each group the trained site showed significant enhancement relative to the contralateral control site (for tail, midbody, and head, $t_{3}$ $=4.12,3.11$, and 5.60, respectively; $p<0.05$ in each case). Since the tentacles lacked the suture markers used at the other sites and showed site-specific sensitization, the site-specific sensitization observed at the other sites does not depend upon the marking procedure.

\section{Week-long enhancement of siphon withdrawal by brief tail stimulation}

To examine more closely the site-specific sensitization of reflex responses, tail stimulation was used as the training stimulus. In an initial $4 \mathrm{~d}$ study it was found that, $2 \mathrm{hr}$ and $4 \mathrm{~d}$ after the 45 


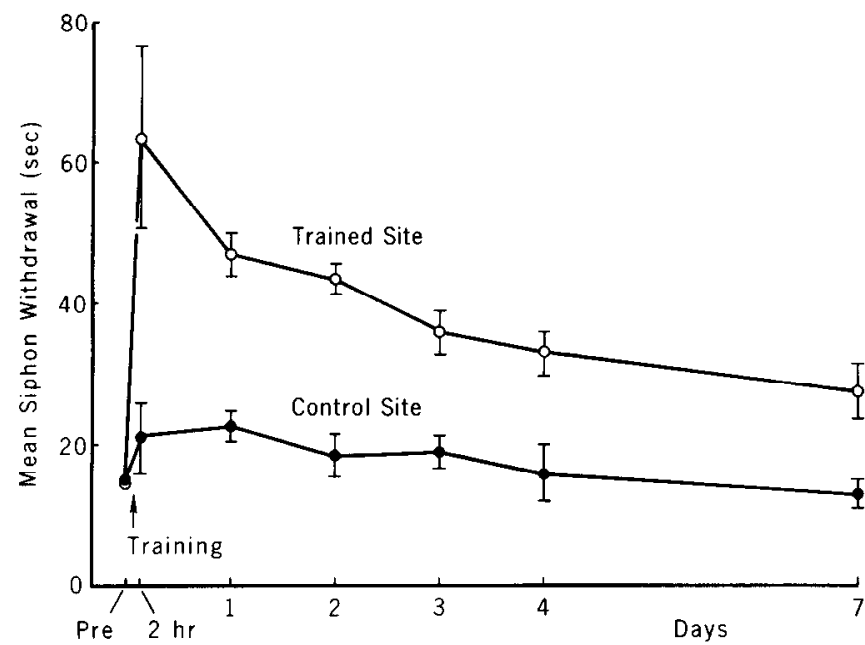

Figure 2. Site-specific sensitization of siphon withdrawal can last a week. The mean $( \pm$ SEM) duration of siphon withdrawal in response to weak shock of bilateral test sites on the tail is shown before and after training one of the sites with the $45 \mathrm{sec}$ sequence of strong shock. The relative enhancement of responses from the trained site demonstrates the long-lasting, site-specific sensitization.

sec training procedure to 1 side of the tail, the siphon responses elicited by testing the trained site were significantly enhanced relative to the responses elicited at the untrained contralateral site $t_{7}=2.57, p<0.05$ and $t_{7}=5.33, p<0.005$, 2-tailed, respectively). A week-long study was then set up with 6 animals from a single shipment, matched by weight and baseline siphon responses (Fig. 2). Two animals were dropped from the experiment, 1 because it failed to release ink during training and 1 because it laid eggs during the retention phase. Two hours after training, the trained site showed a large mean increase in duration of the siphon-withdrawal response $(450 \%$ of the baseline response). The control site also displayed some enhancement ( $140 \%$ of baseline), suggesting the presence of some general sensitization at this timc, although it was not statistically significant. Responses to testing the trained site were significantly greater than responses to the control site $\left(t_{3}=2.69, p<0.05\right)$, indicating that site-specific enhancement was greater than enhancement due to general sensitization $2 \mathrm{hr}$ after training. Seven days after training, the trained site still evoked significantly longer siphon responses than did the control site $\left(t_{3}=3.02, p<0.05\right)$.

\section{Week-long enhancement of the tail-withdrawal reflex}

Because the neural circuit linking tail stimulation to tail withdrawal is known in some detail (Walters et al., 1983a), the tailwithdrawal response was examined to see if it also expressed long-term, site-specific changes following the brief training sequence. Although it is difficult to quantify the tail-withdrawal response in the intact, unrestrained animal, a crude estimate of training-induced changes was obtained by having the "blind" observer ratc the magnitude of cach tail withdrawal on an arbitrary 5-point scale, ranging from no response to a total withdrawal. Figure 3 illustrates the tail-withdrawal responses of the animals whose siphon-withdrawal responses are shown in Figure 2. At $2 \mathrm{hr}$ the mean response from the trained site was greater than the response from the untrained site, but this difference was not significant. At 1 week, however, the trained site evoked significantly greater tail withdrawals than did the untrained site $\left(t_{3}=3.46, p<0.025\right)$. In a separate pilot experiment,

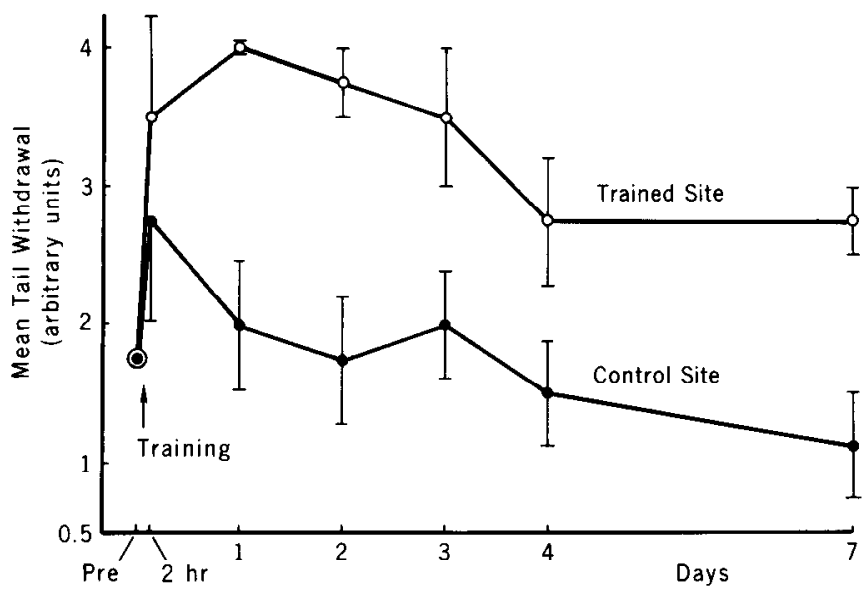

Figure 3. Site-specific sensitization of tail withdrawal can last a week. The magnitude of tail withdrawal (mean \pm SEM) in response to weak shock of bilateral test sites on the tail was estimated in a blind procedure using an arbitrary 5-point scale.

in which the responses were measured for $4 \mathrm{~d}$, the tail withdrawals in response to the trained site were significantly greater than the responses to the untrained site at both $2 \mathrm{hr}$ and $4 \mathrm{~d}$ $\left(t_{7}=2.50\right.$ and 2.32, respectively, $p<0.05$ in each case, 2-tailed).

\section{Site-specific sensitization produced by injury}

Strong electrical shock produces an ensemble of defensive responses that appears identical to that produced by strong mechanical stimulation or cutaneous injury (Walters and Erickson, 1986). Therefore, it seemed likely that the site-specific enhancement produced by shock reflected mechanisms that would normally be activated by injury (produced, for example, by the bite of a predator). To test this idea the same testing procedures were used, but a mechanical "bite" about $1 \mathrm{~cm}$ from the test site was substituted for the strong electrical training stimulus to the test site. The "bite" was applied with a pair of toothed forceps (width $7 \mathrm{~mm}$ ) and consisted of 10 pinches delivered at $5 \mathrm{sec}$ intervals to a site between the suture test marker and the margin of the foot. The separation between test site and training site was small enough to insure that some of the VC sensory neurons' receptive fields would encompass both sites (Walters et al., 1983a) but large enough to prevent damage of the test site. In order to mimic an effective predatory bite more accurately, and to cause unambiguous injury, on the last pinch the tissue was gripped tightly and a piece about $1 \mathrm{~cm}^{2}$ was torn from the side of the tail. Animals that lost the suture marker during or shortly after this noxious "training" procedure were excluded from analysis. The results are illustrated in Figure 4. The subsequent test responses showed considerable variability within each group and displayed a large positive skew. Some of this variability may reflect variation in proximity of the injury to the test marker and variation in the amount of damage done to pathways from the injured site to the CNS. Because of the skew, nonparametric statistics were used, and some of the variability between sides was reduced by normalizing scores to the baseline test for each side in each animal. The basic effect was similar to that produced by strong tail shock: At both 2 hr and $4 \mathrm{~d}$ the siphon withdrawals elicited by stimulating the test site close to the injury were significantly enhanced compared to the responses elicited from the intact, contralateral side of the tail $(N-9, p<0.025$ and 0.01 , respectively, Wilcoxon's signed-rank test). Thus, site-spe- 


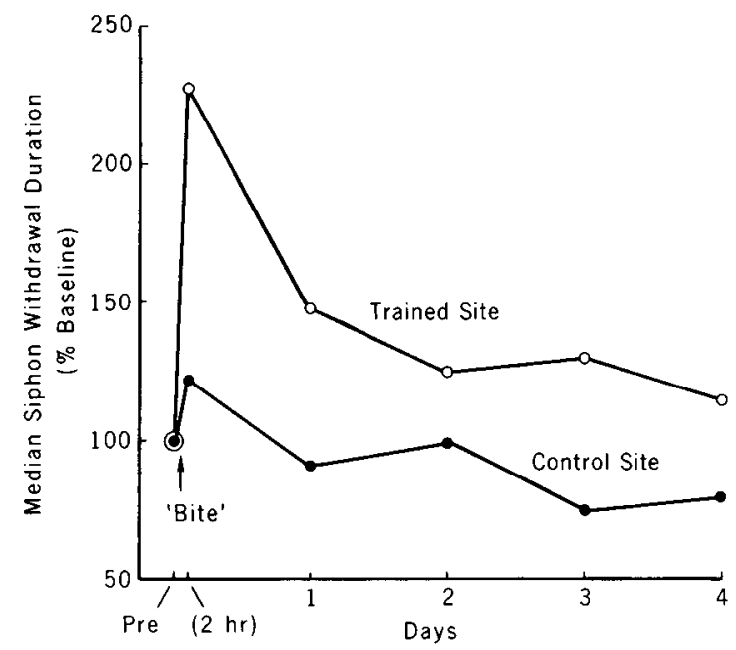

Figure 4. Site-specific sensitization produced by injury. The median duration of siphon withdrawal in response to weak shock of bilateral test sites on the tail is shown before and after training the region adjacent to 1 of the sites with a $45 \mathrm{sec}$ sequence of strong "bites" with toothed forceps. With the last "bite" a small piece of tissue was torn from the side of the tail to insure that a penetrating wound was produced. The range of responses at $2 \mathrm{hr}$ and $4 \mathrm{~d}$, respectively, was as follows: trained site, $81-944 \%$ and $63-261 \%$; control site, $46-192 \%$ and $36-138 \%$.

cific sensitization can be produced by physical injury as well as by electric shock.

\section{Insufficiency of peripheral effects in producing long-term reflex enhancement}

Two studies were performed to examine the possibility that peripheral changes alone might underlie the development and retention of long-term enhancement of defensive reflexes. First, using isotonic $\mathrm{MgCl}_{2}$ for anesthesia, the nerves ( $\mathrm{Lp} 9$ and $\mathrm{Rp} 9$ ) directly connecting the tail to the CNS were transected $3 \mathrm{~d}$ before applying the testing and electrical training procedures described above. Following each experiment the transections were confirmed surgically. The transections reduced the apparent intensity of both the siphon- and tail-withdrawal responses to the test stimuli. Because the siphon withdrawals could be measured more accurately, only the siphon-withdrawal data are presented here. Similar experiments on tail withdrawal utilizing direct measurements of tension are presented in the following paper (Walters, 1987). Figure 5 (top) shows the responses to testing the trained and untrained sites following nerve transection. The pretraining baseline tests produced siphon withdrawals that were shorter than those produced in the intact animal (cf. Fig. 2). A direct comparison of responses in intact and nerve-transected animals cannot be made, however, because the responses were qualitatively different. The siphon responses in the nerve-transected animals were always incomplete, with the siphon never withdrawing fully beneath the parapodia. Therefore, our criterion for siphon-withdrawal duration in these animals was the time taken for the siphon to begin relaxing (reverse direction) after contracting in response to the test stimulus (Pinsker et al., 1973), rather than the time taken to reappear from beneath the parapodia. These contractions were primarily a "funneling" response that may have been secondarily elicited by parapodial movements in response to tail stimulation, rather than the typical "leveling" response produced by tail stimulation in the intact animal (Walters and Erickson, 1986). Training also pro-

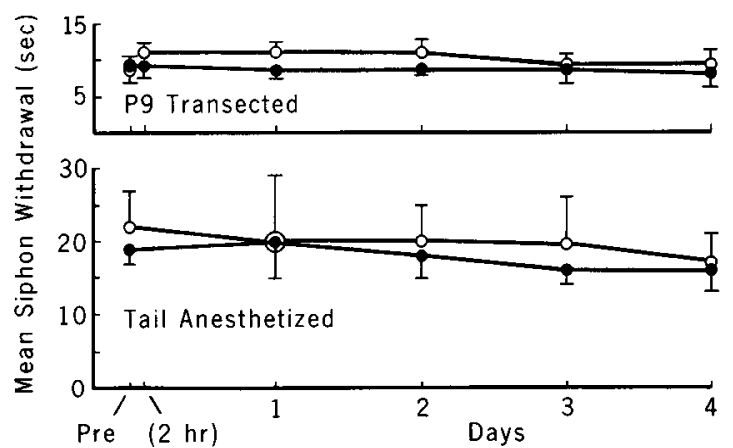

Figure 5. Peripheral effects cannot explain long-term reflex enhancement. The top part of the figure shows the mean $( \pm$ SEM) siphon withdrawals in response to weak shock of the trained site (open circles) and control site (filled circles) before and after the training sequence of strong shock in animals that had previously had the nerves (p9) connecting the tail to the CNS transected. The bottom part shows the results of a similar experiment, but instead of transecting the nerves to the CNS, the stimulation site was anesthetized with isotonic $\mathrm{MgCl}_{2}$ briefly during training. This blocked neural activation while allowing any purely physical effects of the strong training shock to be produced at the training site.

duced different responses in the nerve-transected animals: Only 2 out of 9 animals inked, and there were no obvious locomotor responses to the strong tail shock. In contrast, over $90 \%$ of the intact animals inked and showed rapid locomotor responses to strong electrical or mechanical stimulation of the tail. The test responses following training showed only a slight enhancement relative to baseline, and at both $2 \mathrm{hr}$ and $4 \mathrm{~d}$ there was no significant difference between the siphon responses elicited by testing the trained site and the responses from testing the contralateral control site $\left(t_{8}=0.94\right.$ and 0.80 , respectively). Although there was a hint of a difference at 1 and $2 \mathrm{~d}$ (Fig. 5), these differences were not statistically significant, suggesting that longterm peripheral sensitization in this system is weak at best. The lack of significant site-spccific sensitization was not due to nonspecific effects of the operation since sham-operated animals showed $4 \mathrm{~d}$ site-specific enhancement $\left(t_{4}=2.85, p<0.05\right)$. Therefore, the PNS alone appears insufficient for mediating significant, site-specific, long-term enhancement of the siphon response to tail stimulation.

Failure to observe enhancement of defensive reflexes with the tail functionally isolated from the CNS does not prove that peripheral changes are unimportant. For example, the strong training stimulus might damage the skin, and because of this damage the sensory receptors might become more accessible to subsequent test stimulation. To test the possibility that longlasting physical effects of strong electrical stimulation might explain the long-term enhancement, immediately before training the side of the tail to be trained was injected with $10-20 \mathrm{ml}$ of isotonic $\mathrm{MgCl}_{2}$ to block axonal conduction (as well as generator potentials and synaptic transmission). The $\mathrm{MgCl}_{2}$ solution blocks activation of tail sensory neurons (unpublished observations) and presumably acts by replacing the $\mathrm{NaCl}$ (eliminating $I_{\mathrm{Na}}$ ), increasing firing threshold, and blocking $I_{\mathrm{Ca}}$. The effectiveness of this injection in preventing neural activation was indicated in each case by the lack of behavioral responses (local withdrawal, inking, or locomotion) to the strong training shock. In several cases a fading of the color of the skin was observed at the site that had received the strong shock, but, 
as with uninjected animals, no open wounds or obvious lesions were seen after the strong shock. Because of the apparent persistence of local anesthetic effects of the $\mathrm{MgCl}_{2}$ at $2 \mathrm{hr}$ in some animals, the first test after training was delayed until $1 \mathrm{~d}$ in this study (Fig. 5, lower panel). At both 1 and $4 \mathrm{~d}$ there was no significant difference between the responses to testing the trained and the untrained sites on the tail $\left(t_{7}=-0.97\right.$ and -0.41 , respectively). Thus, any long-lasting physical cffects of the strong electrical training stimulus on the skin are unlikely to cause long-term enhancement of the siphon response to tail stimulation. Although these results are suggestive, it remains possible that the injections of isotonic $\mathrm{MgCl}_{2}$ blocked long-term enhancement by actions other than those on neural activation. For example, the training shock might have been less damaging under local anesthesia because of differences in the amount of tissue stimulated or in the passive electrical characteristics of the stimulated tissue in body wall that had been relaxed by the $\mathrm{MgCl}_{2}$ solution.

These experiments suggest that peripheral changes alone are insufficient to produce site-specific sensitization measured $2 \mathrm{hr}$ to $4 \mathrm{~d}$ after strong tail shock. They do not, however, exclude a peripheral contribution to site-specific sensitization (see following paper).

\section{Long-term enhancement from pairing weak stimulation with strong stimulation of another site}

If ADEM of mechanosensory neurons (Walters and Byrne, 1983, 1985 ) is a mechanism of long-term, site-specific sensitization, then one would predict that this enhancement could be produced by pairing weak, noninjurious stimulation (which activates sensory neurons in the test pathway but by itself does not causc general sensitizing neuromodulation) with strong, noxious stimulation (which causes the diffuse release of sensitizing neuromodulators but not activation of sensory neurons in the test pathway). If the strong stimulus were applied to a site distant from the site of weak stimulation, alteration of responses from the latter site would show, moreover, that local tissue damage is not necessary for the expression of long-term enhancement at a test site. These predictions were tested by pairing weak tail stimulation (too weak to cause sensitizing neuromodulation but strong enough to activate tail sensory neurons; Walters et al., 1983a) with strong electrical stimulation of the head (which causes general sensitizing neuromodulation but no activation of tail sensory neurons; Walters et al., 1983a). This pairing can be considered a classical conditioning procedure, with the weak tail stimulus the conditioned stimulus (CS) and the strong head stimulus the unconditioned stimulus (US). One difference from most classical conditioning procedures is that the CS and US were applied simultancously (ten $0.5 \mathrm{sec}$ trains of $60 \mathrm{~Hz} \mathrm{AC}$ shock were delivered at $5 \mathrm{sec}$ intervals to each site, $45 \mathrm{sec}$ total stimulation). In order to assess the effects of pairing and to compare the results to other conditioning studies, 3 control procedures were run. Within each animal given the paired training an unpaired weak stimulus (45 sec long) was delivered either $5 \mathrm{~min}$ before or $5 \mathrm{~min}$ after the pairing. Thus, these animals underwent a form of differential conditioning. In 2 separate groups of animals the effects of applying the CS alone and the US alone were examined. The test stimulus used in all animals before and after training was a single $0.5 \mathrm{sec}$ train of AC shock, in contrast to the CS used in training the paired, unpaired, and CS-alone groups, which was ten $0.5 \mathrm{sec}$ trains of the same weak shock.
Figure 6 shows the results, which were analyzed with nonparametric statistics because of a large positive skew in the paired and unpaired groups' responses. At $2 \mathrm{hr}$ a Kruskal-Wallis test indicated an overall significant effect of training among the paired, US-alone, and CS-alone groups. Subsequent betweengroup comparisons using the Mann-Whitney $U$ test (2-tailed) indicated that the responses of the paired group $(n=11)$ were significantly greater than those of the US-alone group $(n=8$; $U=19.5 ; p<0.05)$ and CS-alone group $(n=9 ; U=23 ; p<$ 0.05 ). Within-animal comparisons between the paired and unpaired sites using Wilcoxon's signed rank test did not reveal a significant difference between the paired and unpaired sites in this early test ( $n=11, T=26)$. The unpaired group displayed significantly greater responses than the US-alone and CS-alone groups at $2 \mathrm{hr}\left(U_{11,8}=14 ; p<0.02\right.$; and $U_{11,9}=23 ; p<0.05$, respectively). The amount of enhancement at the unpaired site was not significantly different in animals that received the unpaired CS 5 min before the US $(n=6$, median $=316 \%)$ and animals receiving the unpaired CS 5 min after the US $(n=5$, median $=478 \%$ ). At $4 \mathrm{~d}$ a Kruskal-Wallis test again indicated a significant overall effect of training among the paired, USalone, and CS-alone groups. Similarly, the paired group's responses were significantly greater than the US-alone group's $\left(U_{11,8}=8.5 ; p<0.01\right)$ and the CS-alone group's $\left(U_{11,9}=3 ; p<\right.$ 0.001 ). In contrast to the effect at $2 \mathrm{hr}$, Wilcoxon's signed-rank test showed that at $4 \mathrm{~d}$ the paired group had significantly greater responses than the unpaired group ( $n=11, T=9 ; p<0.05$ ). Again, the unpaired group's responses were greater than the responses of the US-alone and CS-alone groups (respectively, $U_{11,8}=18 ; p<0.05$ and $U_{11,9}=20 ; p<0.05$ ). Thus, both the paired and unpaired prescntations of the CS and US cause enhancement relative to the effects of the CS or US alone, both at $2 \mathrm{hr}$ and at $4 \mathrm{~d}$. Although the significant unpaired effects show that the associative enhancement is not completely temporally specific, the significantly greater responses at the paired site than the unpaired site at $4 \mathrm{~d}$ show that there is partial temporal specificity. This temporally specific component of the enhancement is operationally equivalent to a classically conditioned memory.

\section{Discussion}

These results show that brief, noxious stimulation of the skin of Aplysia can produce site-specific sensitization that is retained for at least a week. This site-specific memory is interesting for several reasons. First, site-specific sensitization in Aplysia displays notable similarities to mammalian models of hyperalgesia. Second, site-specific sensitization may be a functionally defined example of a general neural phenomenon - pathway-specific enhancement. Third, the present results are consistent with the possibility that at least part of the mechanism underlying sitespecific memory may be ADEM. Fourth, the possibility that site-specific sensitization involves ADEM of mechanosensory neurons supports the view that some forms of classical conditioning evolved from mechanisms of sensitization.

\section{Similarity to mammalian models of hyperalgesia}

In humans, injury of the skin often causes hyperalgesia-a reduction in threshold and an enhancement in intensity of pain evoked by subsequent cutaneous stimulation. Such hyperalgesia is usually site specific, being restricted to the region surrounding the damaged tissue and can last for weeks after the injury (Lewis, 1942; Hardy et al., 1952). Although pain cannot be studied 
directly in animals because of its subjective component, the similarity of objective responses to injurious stimulation in many animals and in man (e.g., withdrawal, arousal, reflex enhancement) has led to the development of animal models of pain and nociception. A popular preparation has been the mammalian flexion reflex, a response serving to withdraw the limb from a potentially damaging stimulus. Recently, Woolf(1984) has shown that cutaneous injury can cause site-specific cnhancement of flexion reflexes in decerebrate rats that can last for several weeks. This "hyperalgesia," like the site-specific sensitization in Aplysia, involves large increases in both the magnitude and duration of the reflex response to test stimulation. It is also associated with a significant decrease in threshold. Threshold changes have not yet been systematically examined in Aplysia, but during the experiments described in the following paper (Walters, 1987), I sometimes observed the appearance of tail-withdrawal responses to previously subthreshold stimulation following noxious stimulation of the test site. In addition to these outward similarities between objective features of hyperalgesia and sitespecific sensitization in Aplysia, there are also interesting parallels at the neural level that will be described in the following paper (Walters, 1987).

The degree to which site-specific sensitization (and hyperalgesia) is an interesting model for memory depends largely on whether persistent central alterations contribute to site-specific behavioral changes. Although peripheral alterations also make a contribution (Walters, 1987), the present results suggest that central changes are quite important in Aplysia for site-specific reflex enhancement observed $2 \mathrm{hr}$ to 1 week following strong stimulation. More conclusive evidence for central changes contributing to site-specific sensitization is described in the accompanying paper (Walters, 1987).

\section{Similarity to other forms of pathway-specific enhancement}

To my knowledge, the present report is the first to use the term "site-specific sensitization," although the phenomenon is formally similar to a number of other modifications reported at both the behavioral and neuronal levels. Site-specific sensitization probably accounts for the choice made by Aplysia in a conflict situation following prior noxious shock (Walters et al., 1978) and contributes to the increase in tail withdrawal observed previously with repeated moderate tail shock (Walters et al., 1983b). Razran (1971) classifies as "incremental sensitization" cases where repetition of a stimulus causes response enhancement. With strong cutaneous stimulation this enhancement is likely to involve site-specific sensitization and primary hyperalgesia. Indccd, the initial scnsitization often obscrved during habituation, which has usually been interpreted as reflecting a general change in "state" of the organism (Groves and Thompson, 1970; Peeke and Petrinovich, 1984), may in fact have a site-specific component. One can generalize this view to noncutaneous stimuli and predict that sufficiently intense or significant stimuli may sometimes cause nonassociative, stimulusspecific sensitization. Finally, the features of pathway specificity, induction by brief stimulation, and long persistence that are characteristic of site-specific sensitization appear similar to features of LTP in mammals. Because LTP has been described in structures involved in sensory processing (e.g., Gerren and Weinberger, 1983), and even the hippocampus (where LTP has been most extensively examined) is thought to be involved in higher-order sensory processing related to "context" (e.g., Kubie and Ranck, 1984), it is attractive to propose long-term, stim-

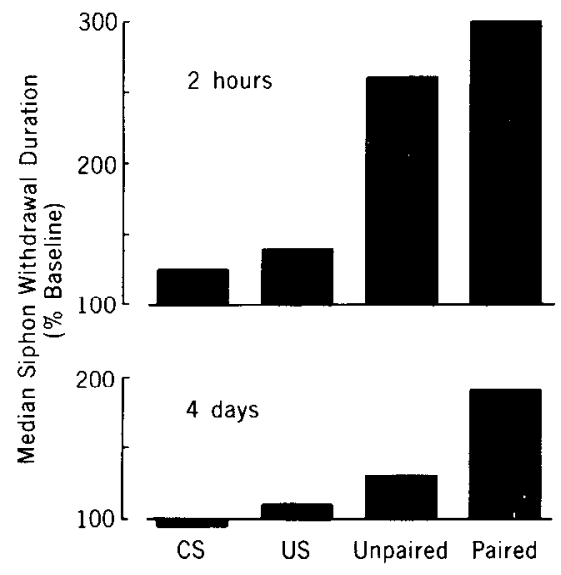

Figure 6. Spatial separation of sensory activation and neuromodulatory components of the training stimulus does not prevent long-term enhancement at the site of activation. A form of differential classical conditioning was used to begin testing the hypothesis that ADEM of sensory neurons in the trained pathway underlies long-term, site-specific sensitization. The conditioned stimulus (CS) was a $45 \mathrm{sec}$ sequence of weak tail shock sufficient to activate identified tail sensory neurons (Walters et al., 1983a) but not strong enough to cause general sensitizing neuromodulation. The unconditioned stimulus (US) was a $45 \mathrm{sec}$ sequence of strong shock to the head that caused general sensitizing neuromodulation but not activation of the identified tail sensory neurons (Walters et al., 1983a). The median siphon withdrawals in response to $0.5 \mathrm{sec}$ test stimuli to the tail are shown for tests conducted $2 \mathrm{hr}$ and 4 $\mathrm{d}$ after training with the CS alone, US alone, unpaired CS and US (separated by $5 \mathrm{~min}$ ), or paired CS and US (applied simultaneously). The range of responses at $2 \mathrm{hr}$ and $4 \mathrm{~d}$, respectively, was as follows: CS, 83-409\% and 73-136\%; US, 32-672\% and 49-278\%; Unpaired, 74$1304 \%$ and $79-387 \%$; Paired, $89-1957 \%$ and $124-1875 \%$.

ulus-specific sensitization as one of probably many functions of LTP (see also Walters and Byrne, 1985).

Long-term, site-specific sensitization of siphon withdrawal in Aplysia is distinct from long-term, general sensitization of siphon withdrawal (Pinsker et al., 1973; Frost et al., 1985). The latter was produced by training stimulation applied at a distance from the test site and involved the "spacing" of training stimuli over days or hours rather than "massing" them into less than a minute. Long-term, general sensitization seems unlikely to involve pathway-specific mechanisms.

\section{Possible involvement of ADEM in identified sensory neurons}

Both electrical and mechanical stimulation of the tail activate tail sensory neurons in the VC cluster of the pleural ganglion (Walters et al., 1983a). Noxious or damaging stimuli are particularly effective, eliciting bursts of action potentials ranging from 20 to $80 \mathrm{~Hz}$ (Walters et al., 1983a, and unpublished observations). Therefore, both the $60 \mathrm{~Hz}$ shocks and mechanical stimuli used for cutaneous training should have activated these cells and activated them at frequencies similar to those used previously to produce ADEM and long-term enhancement of VC sensory neurons (Walters and Byrne, 1983, 1985). Indeed, since the same stimulation pattern used for cutaneous training in the present study was previously shown to cause long-term enhancement of sensory neuron connections when applied to the nerve containing the sensory neuron axons (Walters and Byrne, 1985), it seems likely that skin stimulation in the intact animal causes both the behavioral effects described here and the cellular effects reported earlier. Similar conclusions emerge from the following paper (Walters, 1987), which describes site-specific 


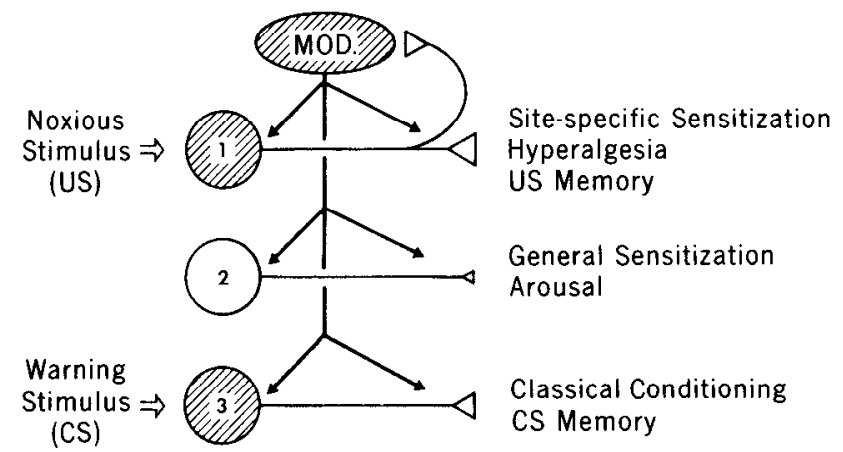

Figure 7. Model of somatosensory plasticity during noxious stimulation. A noxious unconditioned stimulus (US) activates wide-dynamicrange mechanosensory neurons (cell 1 ), which in turn activate the neuromodulatory system $(M O D)$ and cause the diffuse release of sensitizing neuromodulators (arrows). In mechanosensory neurons that are inactive at the timc of the US (cell 2), the neuromodulators cause transient, weak enhancement of synaptic release and of cell excitability, transiently increasing the cells' signaling effectiveness. These widespread effects contribute to general behavioral sensitization and defensive arousal. By contrast, cells that are active at the time that the sensitizing neuromodulators are released (shading) undergo ADEM, with the intrinsic spike activity amplifying the degree and duration of enhancement produced by extrinsic neuromodulators. This pairing occurs in 2 pathways, represented by cell 3 , mediating a warning CS (if present), and cell 1 , mediating the noxious US. ADEM in the CS pathway produces a memory of the CS, and the same mechanism in the US pathway produces a trace of the noxious US that functionally resembles hyperalgesia.

cellular changes produced by noxious cutaneous shock in a semiintact preparation.

Although the present study did not directly examine the possible involvement of ADEM at the cellular level, it did provide some behavioral evidence suggestive of a role for this mechanism in site-specific sensitization. First, blocking activation of the sensory neurons by injecting isotonic $\mathrm{MgCl}_{2}$ at the training site prevented reflex enhancement (Fig. 5). Second, neither activation alone nor neuromodulation alone appears to be sufficient to produce long-term, site-specific sensitization in the intact animal. This was suggested by the failure of the weak CS to the tail (which does not produce general sensitization-a behavioral index of general neuromodulation) and the strong US to the head (which produces brief general sensitization but not activation of the tail sensory neurons; Walters et al., 1983a) to produce long-term reflex enhancement (Fig. 6). Finally, when weak tail stimulation (sensory neuron activation) was paired with neuromodulation produced by distant sensitizing stimulation, long-term, site-specific sensitization of tail-elicited reflexes occurred (Fig. 6).

\section{Relationships among sensitization, site-specific sensitization, and classical conditioning}

Wells (1968) and Razran (1971) suggested independently that behavioral sensitization may have been an evolutionary precursor of associative learning. This possibility was strengthened recently by indications that some forms of associative conditioning utilize cellular mechanisms involved in general sensitization (Hawkins et al., 1983; Walters and Byrne, 1983; Livingstone et al., 1984), and Hawkins and Kandel (1984) have suggested that a small change in adenylate cyclase in the sensory neurons could underlie this evolution. The present discovery of site-specific sensitization in a preparation that shows both general sensitization and classical conditioning and the evidence for a role of ADEM in this site-specific sensitization (Walters, 1987) support these views and raise the possibility that sitespecific sensitization was an intermediate link between general sensitization and classical conditioning. Two inferences about ADEM-mediated plasticity in the somatosensory system of Aplysia (Fig. 7) suggest that ADEM may have evolved initially for site-specific sensitization and later been adapted for classical conditioning. First, ADEM and site-specific sensitization should occur in a US pathway whenever cutaneous classical conditioning occurs by ADEM of a CS pathway (Fig. 7). However, since noxious cutaneous USs can also occur in the absence of any cutaneous warning CS, more opportunities will have arisen during evolution for the natural selection of site-specific sensitization than for classical conditioning. Second, for cutaneous classical conditioning to be advantageous, there should be a high probability that subsequent encounters with the source of the US will be signaled by the CS. However, cutaneous sensory neurons have rather small receptive fields (Byrne et al., 1974; Walters et al., 1983a). Consequently, the probability that a predator's warning touch will be delivered to exactly the same region of skin that was stimulated in the original (conditioning) attack is not very high. If a warning CS is delivered outside the conditioned receptive fields, the previous learning by this mechanism will provide no advantage.

Site-specific sensitization and cutaneous hyperalgesia (Fig. 7) are probably important for protecting an injured site from further stress. Not only are injured sites likely to be weakened physically; they are liable to additional attack from predators and parasites: Some substances that leak from wounds can act as chemoattractants (e.g., Lapage, 1958; Bardach et al., 1959), and other characteristics of an injury can attract predatory attention (Curio, 1976). Although general defensive sensitization (Fig. 7) will serve to protect an injury in the short term by enhancing defensive reactions and suppressing spontaneous movements, it will not be economical for an animal to maintain general defensive arousal over the long periods required for a serious injury to heal. Site-specific sensitization allows the animal to resume normal behavior (e.g., feeding and mating), while still maintaining enhanced responsiveness in the injured region. Finally, during classical conditioning, site-specific sensitization within a US pathway will result in a memory of the US produced in parallel with the memory of the CS in the CS pathway (Fig. 7). Central traces produced by such mechanisms may contribute to the "internal representations" of the US some believe to be important for associative information processing (e.g., Tolman, 1932; Konorski, 1967).

\section{References}

Bardach, J. E., H. E. Winn, and D. W. Menzel (1959) The role of the senses in the feeding of the nocturnal reef predators Gymnothorax moringa and G. vicinus. Copeia 1959: 133-139.

Berger, T. W. (1984) Long-term potentiation of hippocampal synaptic transmission affects rate of behavioral learning. Science 224:627630.

Byrne, J., V. Castellucci, and E. R. Kandel (1974) Receptive fields and response properties of mechanoreceptor neurons innervating siphon skin and mantle shelf in Aplysia. J. Neurophysiol. 37: 10411064.

Carew, T. J., E. T. Walters, and E. R. Kandel (1981) Classical conditioning in a simple withdrawal reflex in Aplysia californica. J. Neurosci. $I: 1426-1437$.

Carew, T. J., R. D. Hawkins, and E. R. Kandel (1983) Differential classical conditioning of a defensive withdrawal reflex in Aplysia californica. Science 219: 397-400.

Castellucci, V., H. Pinsker, I. Kupfermann, and E. R. Kandel (1970) 
Neuronal mechanisms of habituation and dishabituation of the gillwithdrawal reflex in Aplysia. Science 167: 1745-1748.

Curio, E. (1976) The Ethology of Predation, Springer-Verlag, Berlin.

Frost, W. F., V. F., Castellucci, R. D. Hawkins, and E. R. Kandel (1985) Monosynaptic connections made by the sensory neurons of the gilland siphon-withdrawal reflex in Aplysia participate in the storage of long-term memory for sensitization. Proc. Natl. Acad. Sci. USA 82: 8266-8269.

Gerren, R. A., and N. M. Weinberger (1983) Long term potentiation in the magnocellular medial geniculate nucleus of the anesthetized cat. Brain Res. 265: 138-142.

Groves, P. M., and R. F. Thompson (1970) Habituation: A dualprocess theory. Psychol. Rev. 77: 419-450.

Hardy, J. D., H. G. Wolff, and H. Goodell (1952) Pain Sensations and Reactions, Hafner, New York.

Hawkins, R. D., and E. R. Kandel (1984) Is there a ccll-biological alphabet for simple forms of learning? Psychol. Rev. 91: 375-391.

Hawkins, R. D., T. W. Abrams, T. J. Carew, and E. R. Kandel (1983) A cellular mechanism of classical conditioning in Aplysia: Activitydependent amplification of presynaptic facilitation. Science 219:400404.

Ingram, D. A., and E. T. Walters (1984) Differential classical conditioning of tail and siphon withdrawal in Aplysia. Soc. Neurosci. Abstr. 10:270.

Konorski, J. (1967) Integrative Activity of the Brain: An Interdisciplinary Approach, University of Chicago Press, Chicago, IL.

Kubie, J. L., and J. B. Ranck (1984) Hippocampal neuronal firing, context, and learning. In Neuropsychology of Memory, L. R. Squire and N. Butters, eds., pp. 417-423, Guilford, New York.

Lapage, G. (1958) Parasitic Animals, Cambridge University Press, Cambridge, England.

Levy, W. B., and O. Steward (1983) Temporal contiguity requirements for long-term associative potentiation/depression in the hippocampus. Neuroscience 8: 791-797.

Lewis, T. (1942) Pain, Macmillan, New York.

Livingstone, M. S., P. P. Sziber, and W. G. Quinn (1984) Loss of calcium/calmodulin responsiveness in adenylate cyclase of rutabaga, a drosophila learning mutant. Cell 37: 205-215.
Peeke, H. V. S., and L. Petrinovich. (1984) Habituation, Sensitization, and Behavior, Academic, New York.

Pinsker, H. M., W. A. Hening, T. J. Carew, and E. R. Kandel (1973) Long-term sensitization of a defensive withdrawal reflex in Aplysia. Science 182: 1039-1042.

Razran, G. (1971) An East-West Synthesis of Learned Behavior and Cognition, Houghton Mifflin, Boston, MA.

Snedecor, G. W., and W. G. Cochran (1980) Statistical Methods, Iowa State University Press, Ames, IA.

Tolman, E. C. (1932) Purposive Behavior in Animals and Men, Appleton-Century-Crofts, New York.

Walters, E. T. (1985) Site-specific sensitization in Aplysia, LTP, regenerative bursting, and a possible link in the evolution of learning. Soc. Neurosci. Abstr. 11: 795.

Walters, E. T. (1987) Multiple sensory neuronal correlates of sitespecific scnsitization in Aplysia. J. Neurosci. 7: 408-417.

Walters, E. T., and J. H. Byrne (1983) Associative conditioning of single sensory neurons suggests a cellular mechanism for learning. Science 219: 405-408.

Walters, E. T., and J. H. Byrne (1985) Long-term enhancement produced by activity-dependent modulation of Aplysia sensory neurons. J. Neurosci. 5: 662-672.

Walters, E. T., and M. T. Erickson (1986) Directional control and the functional organization of defensive responses in Aplysia. J. Comp. Physiol. 159: 339-351.

Walters, E. T., T. J. Carew, and E. R. Kandel (1978) Conflict and response selection in the locomotor system of Aplysia. Soc. Neurosci. Abstr. 4: 209.

Walters, E. T., J. H. Byrne, T. J. Carew, and E. R. Kandel (1983a) Mcchanoafferent neurons innervating tail of Aplysia. I. Response properties and synaptic connections. J. Neurophysiol. 50: 1522-1542.

Walters, E. T., J. H. Byrne, T. J. Carew, and E. R. Kandel (1983b) Mechanoafferent neurons innervating tail of Aplysia. II. Modulation by sensitizing stimulation. J. Neurophysiol. 50: 1543-1559.

Wells, M. (1968) Lower Animals, McGraw-Hill, New York.

Woolf, C. J. (1984) Long term alterations in the excitability of the flexion reflex produced by peripheral tissue injury in the chronic decerebrate rat. Pain 18: 325-343. 